\title{
Paysages médiatiques et transformations du joumalisme au Sénégal depuis les années 2000
}

\section{Entretien avec Ousmane Mangane}

\author{
Martin Mourre
}

\begin{abstract}
Citer cet article : Mourre Martin (2021), « Paysages médiatiques et transformations du journalisme au Sénégal depuis les années 2000. Entretien avec Ousmane Mangane ", Revue d'Histoire Contemporaine de l'Afrique, en ligne. URL : https://oap.unige.ch/journals/rhca/article/view/entmourre

Mise en ligne : 7 janvier 2021

DOI : https://doi.org/10.51185/journals/rhca.2021.e301
\end{abstract}

Cet entretien avec le journaliste sénégalais Ousmane Mangane offre l'occasion de prolonger la réflexion initiée dans ce premier numéro de la Revue d'Histoire Contemporaine de l'Afrique portant sur l'histoire des médias sur le continent entre les années 1940 et 1970. Né en 1985 à Lagbar, un village du Ferlo, une région pastorale et très désertique située dans le nord du Sénégal, Ousmane Mangane s'est très tôt impliqué dans la presse scolaire, puis la presse radiodiffusée et télévisée. À travers son parcours, cet entretien est l'occasion de revenir sur plus de vingt ans de journalisme au Sénégal et de suivre la façon dont s'exerce la profession de journaliste aujourd'hui. II éclaire les liens entre journalisme et politique au Sénégal, ainsi que les transformations du secteur des médias. Ousmane Mangane publie son autobiographie intitulée "Carnets de voyage d'un reporter », à paraître dans le courant de l'année 2021 aux éditions Feu de brousse dirigées par le poète Amadou Lamine Sall'1. Cet entretien a été réalisé en octobre 2020 à Dakar puis relu par Ousmane Mangane.

\section{Bonjour Ousmane Mangane. Pourriez-vous revenir sur vos premières expériences comme journaliste au sein de la presse scolaire, un secteur qui est très dynamique au Sénégal ?}

Depuis tout petit j'ai voulu faire du journalisme mais j'ai commencé véritablement quand je suis arrivé au Lycée Seydina Limamou Laye de Guédiayawe, à l'époque le seul lycée de la banlieue de Dakar. II regroupait déjà près de 3000 élèves et c'est aujourd'hui le plus grand lycée du Sénégal, avec des élèves qui viennent de l'ensemble du pays. Quand je suis venu, j'ai aussitôt intégré le club de français, dirigé par Abou Mbow, qui deviendra ensuite le directeur du livre et de la lecture au ministère de la Culture. On avait aussi notre journal du lycée. C'était intéressant car cela permettait de se retrouver entre lycéens sur divers sujets. On échangeait sur des questions sociales, politiques, qui intéressaient l'Afrique. Nous nous exercions à la prise

\footnotetext{
${ }^{1}$ Voir une présentation de l'éditeur en ligne. URL : https://www.printempsdespoetes.com/Les-editions-feu-de-brousse (consulté le 3 janvier 2021).
} 
de parole en public comme à l'écriture d'articles. En avril 2004 a été initié le $1^{\text {er }}$ Festival des journaux lycéens à la Maison de la Culture Douta Seck à Dakar, sous la tutelle de Mme AndréeMarie Diagne ${ }^{2}$. Plusieurs établissements du Sénégal ont participé comme le Prytanée militaire de Saint Louis, les lycées Blaise Diagne, Seydou Nourou Tall, le Lycée International Bilingue et même une délégation brésilienne. L'ouverture de ce premier festival des journaux lycéens, le $1^{\text {er }}$ avril 2004, a été un jour important pour les journalistes en herbe que nous étions. II y avait notamment le doyen Mame Less Camara ${ }^{3}$ parmi les conférenciers et d'autres journalistes assez connus comme El Bachir Sow du Soleil ou Doudou Coulibaly de la Radio Manoré FM (La voix des femmes). Dans le festival, il y avait un jury qui statuait sur ces différents journaux, leurs contenus, l'écriture des articles et qui à la fin décernait un prix, que le Lycée Limamou Laye a d'ailleurs remporté. La presse scolaire a beaucoup contribué à ma formation.

\section{Et après le lycée vous avez souhaité faire des études de journalisme ?}

J'ai d'abord pu effectuer une visite à la Radio Télévision Sénégalaise (RTS) avec les camarades du lycée. Cela m'avait impressionné et cela a confirmé le goût que j'avais pour le journalisme. Après avoir obtenu le Baccalauréat en 2005 j'ai suivi des cours à distance avec «Educatel Rouen », jusqu'en 2009. C'était une formation à distance4, et je pouvais aussi prendre des cours à I'Université Cheikh Anta Diop de Dakar, où j'ai suivi un cursus au département de lettres modernes. J'ai aussi pu réaliser des stages, notamment dans le groupe de presse Walfadjri. Et puis plus tard, en 2016, alors que j'avais déjà commencé à travailler comme journaliste, j'ai repris une formation et j'ai obtenu le «Diplôme Supérieur en Journalisme et Communication, option Communication Institutionnelle » à I'Institut Supérieur des Sciences de I'Information et de la Communication de Dakar (ISSIC). Mon mémoire de Master 2 avait pour titre Diagnostic de la stratégie de communication de la mission médicale chinoise au Sénégal.

\section{Quelles ont été vos premières expériences sur le terrain en tant que reporter?}

Nous étions en 2006-2007, j'ai fait un stage de près de huit mois dans une radio locale, Radio Disso FM, à Mbacké Touba5, à l'intérieur du Sénégal. C'était un choix de ma part d'aller là-bas avant de rejoindre Dakar. Et cela m'a permis de travailler plusieurs petites techniques du métier de journaliste radio avant d'intégrer les grands médias. J'avais choisi d'aller là-bas pour faire un stage et affronter le micro. C'était une radio qui appartenait à un marabout, Sérigne Aziz.

\footnotetext{
2 Docteur en lettre, Mme Andrée-Marie Diagne a été animatrice d'émissions littéraires et d'ateliers d'écriture. Elle a également rédigé plusieurs manuels scolaires du secondaire et un recueil de nouvelles (La Fileuse d'amour, Harmattan Sénégal, 2013). Au Sénégal, elle est également très impliquée dans la promotion des carrières féminines.

3 Journaliste et chroniqueur politique, Mame Less Camara a une longue carrière de journaliste derrière lui. II débute dans les années 1980 à la Radio Télévision Sénégalaise (RTS), et est le premier à diriger la radio Walfadjri avant de devenir le correspondant local de la BBC à Dakar. Il a également formé nombre de journalistes sénégalais, au point que parfois on l'affuble du surnom de "Père Less ». ${ }^{4}$ Sur les enjeux de la formation à distance en Afrique, voir Kane Oumar (2008), "La FAD en Afrique francophone. Éléments historiques et enjeux récents », Distances et savoirs, 6, pp. 69-82. Plus spécifiquement sur ces " e-learning » dans le cas des médias africains, voir Caudron Fabrice, Grecos Myrto, Jeafar Ibtisseme (2018), «La formation des cadres des médias africains par le e-learning. Étude de cas d'un partenariat universitaire au niveau Master », @GRH, 26, pp. 79-102.

5 La ville de Touba est le siège de la confrérie Mouride, une des plus importantes confréries musulmanes au Sénégal, où repose le tombeau de son fondateur, Cheikh Ahmadou Bamba. Elle est souvent surnommée «la ville sainte » du Sénégal.
} 
La radio était dirigée par un grand reporter de Walfadjri, Benjamin Diagne ${ }^{6}$. Le marabout avait décidé de faire comme à Dakar une radio généraliste à Touba et moi j'ai eu l'opportunité de travailler ici, donc c'était une chance dans ma carrière. Cette radio émettait dans la zone de Touba-Mbacké mais également à l'international, via Worldspace ${ }^{7}$. Puis, en 2007, j'ai pu intégrer Walf-FM pour un stage de trois mois. C'était au moment de la présidentielle. Mon premier reportage remonte au 4 février 2007, soit le premier jour de la campagne présidentielle. J'étais avec le candidat Abdoulaye Bathily, membre de l'opposition et qui est par ailleurs un historien reconnu ${ }^{8}$.

\section{Vos premières armes ont donc été réalisées pendant la campagne présidentielle de 2007. Quels souvenirs marquants en gardez-vous ?}

Je faisais deux reportages par jour, un le matin et un pour le journal de midi. Et le soir, je continuais d'accompagner un candidat. Un des événements marquants de la campagne a été l'attaque du cortège du candidat Idrissa Seck $^{9}$ par les proches du marabout Cheikh Béthio Thioune. La journaliste Aissatou Diop Fall qui accompagnait le candidat Idrissa Seck ce jour-là a été exfiltrée rapidement, et puis moi je suis arrivé sur les lieux un peu après avec Mamadou Ndiaye Doss - il présentait le journal sur Walf-TV puis des émissions sur Sen TV; il vient de décéder en mars 2020 et je salue sa mémoire. Pendant toute la campagne, je me trouvais à Dakar mais j'ai couvert le jour de l'élection à Linguère, qui est une ville très politisée. Finalement Abdoulaye Wade - le président sortant - a gagné ici largement.

\section{Walfadjri est un groupe de presse qui a une radio, une télé, un journal. Pourriez-vous revenir sur son histoire?}

Walfadjri est devenu un très grand groupe de presse en 1997. Au début, il n'y avait qu'un journal, Walf Quotidien lancé en 1984, puis une radio, Walf FM, créée en 1997. Au début des années 2000, Walfadjri était composé de plusieurs journaux, Walfadjri, Walfsport et Walf Grand'Place, d'une radio généraliste, Walf- $F M$, deux radios thématiques, Walf 2 qui est une radio religieuse, Walf 3 , qui est une radio musicale - sans compter les stations régionales - et une télévision Walf- TV. II y a eu des restructuration depuis, mais la ligne du groupe est claire : ne pas dépendre des orientations de l'État, couvrir toutes les manifestations où les gens contestent, disent leur point de vue, etc. II s'agit d'ouvrir le micro pour tous les gens qui se sentent lésés. Parce qu'avant, c'était le parti unique, et la pensée semblait être unique. Il y avait

\footnotetext{
${ }^{6}$ Benjamin Diagne est un personnage aux vies multiples, journaliste, poète, écrivain, scénariste et réalisateur sénégalais. Pour une brève biographie, voir en ligne, URL :

http://africultures.com/personnes/?no=16595\&utm_source=newsletter\&utm_medium=email\&utm_campaign=482 (consulté le 3 janvier 2021).

7 Worldspace est un groupe de médias basé aux États-Unis possédant son propre réseau satellitaire et diffusant dans plus d'une centaine de pays.

${ }^{8}$ Abdoulaye Bathily est un historien sénégalais formé à Birmingham dans les années 1970, où il soutient une thèse sur le royaume du Galam, au nord-est du Sénégal, du VIIIème au XVIIIème siècle. C'est un acteur politique important du mouvement étudiant dès les années 1960, où il participe au « mai 68 » sénégalais. En 1974, il fonde la Ligue démocratique, issue d'une scission avec le Parti Africain de l'Indépendance - parti le plus à gauche de l'échiquier politique et dans la clandestinité à cette époque. En 2017, il est candidat à la présidence de I'Union Africaine, où il échoue de peu.

9 Idrissa Seck est l'ancien premier ministre d'Abdoulaye Wade, de 2002 à 2004. En 2007, il se présente contre ce dernier et finit deuxième au premier tour avec un peu plus de 14 \% des voix. II se représente en 2012 puis en 2019, où il obtient 20 \% des voix au premier tour, finissant également derrière Macky Sall crédité quant à lui de plus de $58 \%$ des voix.
} 
bien Sud-FM", mais l'arrivée de Walf-FM a permis de remettre en cause cette pensée et cette voix unique. Quand la radio Walf-FM est née, elle a joué un grand rôle au Sénégal notamment dans les élections présidentielles de $2000^{11}$. Ainsi, dès que les bureaux de vote ont fermé, les résultats commençaient à être donnés à la radio. À cette époque, les téléphones portables commençaient à être accessibles pour les journalistes qui en avaient chacun un et qui donnaient les résultats en direct. C'était différent des élections précédentes où l'on apprenait les résultats par un communiqué. Une des ambitions de Sidy Lamine Niasse, le fondateur de Walfadjri, était de créer un très grand groupe de presse, très puissant. II disait que dans un avenir proche il voulait faire un grand groupe de télévision en partant d'ici, du Sénégal. Donc en 2007, un peu avant les élections, Sidy Lamine Niasse, que l'on surnomme le "Mollah de Sacré Cœur ${ }^{12}$ », a souhaité lancer une chaîne de télévision sur la même ligne éditoriale que la radio. Au début, il n'avait pas l'autorisation de diffuser par la voie hertzienne. Peut-être que I'État a senti que c'était une menace et le gouvernement n'a pas voulu leur donner l'autorisation d'émettre par voie hertzienne et d'être capté un peu partout.

\section{Justement, pouvez-vous dire un mot de la transformation du secteur des médias au Sénégal, avec l'arrivée de nouvelles technologies de l'information?}

Je pense que le changement s'est déroulé avec la création de Walf-TV. En 2007, quand ils auraient dû commencer à diffuser, Sidy Lamine Niasse n'a pas eu l'autorisation d'émettre sur le territoire national et il était obligé de passer par un satellite en France. Niasse s'est tourné vers France Télécom avec qui il a signé un contrat. Et ils ont diffusé par le canal satellite via "Globe Cast » qui était le diffuseur, et qui aujourd'hui diffuse des programmes télé par satellite, numérique, réseaux câblés, etc. Niasse a déboursé près d'un million et demi d'euros pour que la chaîne soit diffusée. Heureusement il y avait déjà un public d'auditeurs fidèles qui a suivi quand Walf-TV s'est lancée. II fallait des antennes paraboliques pour capter les programmes et il y en a eu de plus en plus dans les villages, même s'il fallait acheter une carte d'abonnement, donc c'était un budget aussi. Si la télévision émettait depuis la France, elle était reçue un peu partout en Afrique et en Europe, à destination de la diaspora sénégalaise. Les images étaient envoyées en France avant d'être montées là-bas et réacheminées. Dans un premier temps, le journal était complété par les correspondants régionaux, nous envoyions des reportages audio qui étaient " habillés » par des images sur un plateau fixe à Dakar. Certains disaient de nous que nous étions en train de faire de la "Télé-Radio ». Finalement, les correspondants ont commencé à faire des envois d'images qu'ils filmaient parfois eux-mêmes, parfois avec l'aide d'un caméraman (comme le cadreur Saliou Béye, très connu dans le milieu). Les images n'étaient pas toujours de bonne qualité mais avec ce procédé Walf-TV a révolutionné la télévision en donnant des informations en temps réel. En 2008, Walf-TV est

\footnotetext{
10 Sud-FM est créée au début des années 1990. Elle est, historiquement, la première radio privée du Sénégal.

112000 correspond à l'élection d'Abdoualye Wade après quarante années de pouvoir du parti socialiste. Le journal Walf Quotidien est aussi connu pour avoir été le premier à titrer «Wade président » au lendemain du second tour de l'élection présidentielle.

12 Sidy Lamine Niasse, est issu de la confrérie Niassène, une branche de la Tidjaniya. Né en 1950, il part se former au droit islamique à I'Université Al Azhar au Caire à la fin des années 1970 avant de retourner au Sénégal. Son frère, guide religieux comme lui, avait créé en 1979 un parti politique au Sénégal nommé «le parti de Dieu » à la suite de la révolution iranienne -il sera rapidement interdit. Sacré-Cœur est le nom d'un quartier dakarois dans lequel est installé le groupe de presse.
} 
devenue une télévision très regardée derrière les deux chaînes, RTS et $2 S T V$, puis la plus suivie en 2009.

\section{Pourriez-vous préciser le rôle de Walfadjri par rapport à la scène politique dans les années 2000 ?}

Dans l'opinion, ce que disent les gens, c'est que Walfadjri n'est jamais avec le pouvoir. Il est toujours avec les opposants. Sidy Lamine Niasse disait que quand vous voyez cela, ça rassure. Walf, télé et radio, ouvre le micro aux opposants, aux contestataires, et aussi le groupe a une capacité de mobilisation extraordinaire. Le 25 septembre 2009 les locaux ont été attaqués par des nervis, peut-être recrutés par des membres du pouvoir, avec des dégâts matériels et aussi des gens blessés. C'est arrivé plusieurs fois ensuite pour différentes raisons. Mais si Sidy Lamine Niasse fait une déclaration afin de mobiliser des auditeurs ou des soutiens, c'est une marée humaine qui déferle. Vous pouvez même penser que c'est une campagne électorale. Certains disaient que la capacité de mobilisation de Walfadjri était de beaucoup supérieure à celles de leaders politiques. À titre personnel, j'ai aussi subi une attaque en 2008 en tant que journaliste de Walf-TV. C'était en mars, pendant ce que l'on a appelé les émeutes de la faim. Il y avait une grande marche de protestation, des leaders de l'opposition, comme Talla Sylla ${ }^{13}$, avaient été arrêtés et d'autres s'étaient réfugiés à la permanence du Parti de l'Indépendance et du Travail (PIT), proche du siège de Walf-TV. II y avait la député Mously Diakhaté14 à qui j'ai tendu un micro. C'est le geste qu'il ne fallait pas faire et j'ai aussitôt reçu une décharge électrique par un policier. Nous étions en direct à la télévision, les téléspectateurs ont vu les images comme dans un film. Finalement les manifestants m'ont transporté à l'extérieur de la marche. Cette affaire a eu un grand retentissement, j'ai reçu un appel du comité de protection des journalistes de New York. L'affaire avait été relayée au niveau international. Une lettre a été écrite au Président de la République du Sénégal par cette même association, avec des copies adressées à plusieurs autorités sur le plan mondial en charge des droits humains.

\section{Walfadjri est présenté comme un média engagé mais y-a-t-il d'autres raisons qui expliquent sa popularité au Sénégal, notamment sa couverture des questions religieuses?}

Tout à fait. Sidy Lamine Niasse accordait une importance particulière aux cités confrériques. Walf-TV couvrait pratiquement toutes les manifestations religieuses qui se déroulaient dans ces localités. II y a d'abord le Magal de Touba ${ }^{15}$. II faut noter que Walf-TV a révolutionné la couverture médiatique de ce grand événement. Je peux dire sans risque de me tromper que Walf-TV est la première télévision à faire des éditions en direct depuis Touba. Le premier grand direct a été réalisé avec l'aide d'une équipe d' $A$ / Jazeera basée à Nouakchott. Depuis, le grand Magal est suivi en direct depuis le premier jour, marquant le départ en exil du fondateur du mouridisme Cheikh Ahmadou Bamba, jusqu'à la cérémonie officielle. Le premier direct du

\footnotetext{
13 Talla Sylla est un ancien leader étudiant des années 1980. Candidat sous la bannière de l'Alliance pour le progrès et la justice, il recueille moins d' $1 \%$ des voix. Depuis 2014 il est maire de la ville de Thiès.

14 Mously Diakhaté appartenait au parti de Talla Sylla, avec qui elle rompt en 2012 pour soutenir la coalition présidentielle de Macky Sall.

15 Le Magal de Touba est le grand rassemblement de la confrérie mouride, où est célébré le départ en exil du fondateur de la confrérie, Cheikh Ahmadou Bamba. II peut réunir jusqu'à plus d'un million de fidèles qui se rendent dans la ville sainte de Touba.
} 
journal de midi depuis Touba m'a beaucoup marqué en tant que jeune journaliste. Les reporters étaient installés aux différents coins de la mosquée et il était difficile de faire le direct devant cette foule nombreuse. Certains pèlerins voulaient coûte que coûte apparaître à la télévision et il fallait être courageux pour faire face aux regards des pèlerins à la caméra. De 2007 à 2011, avec l'équipe de la radio et de la télévision, je n'ai pas raté cette manifestation religieuse. Mais il y a aussi les autres cités confrériques, comme l'inauguration de la mosquée de Médina Baye à Kaolack en 2010 ou encore les commémorations à Tivaouane, qu'il s'agisse de la confrérie Tidjiane ou celle des Moustarchidines ${ }^{16}$ que Walf-TV s'est mise à couvrir.

\section{Dans votre autobiographie, vous évoquez aussi la naissance de TFM, qui est un autre grand groupe de presse, lancé par le musicien Youssou N'dour.}

Youssou N'dour était propriétaire d'une radio, Radio Futurs Médias (RFM), et d'un journal, L'Observateur. II a voulu agrandir ce groupe de presse en lançant une télévision. Quelques mois plus tôt il était venu à Walfadjri pour une visite de travail et avait rencontré beaucoup de gens qui y travaillaient. II y a eu une discussion ouverte entre Sidy Lamine Niasse et Youssou N'dour. "Yous » a dit que Walfétait une référence et, qu'en quelque sorte, il cherchait à s'en inspirer. Il disait que cela aurait été des télévisions complémentaires. Et puis deux ans plus tard, Youssou N'dour s'est lancé dans la course à la présidentielle. Beaucoup de journalistes de RFMn'étaient pas pour ce mélange des genres et ont refusé de le soutenir explicitement. J'ai eu l'occasion d'aller travailler temporairement à RFM comme correspondant en 2014, pendant environ onze mois. On m'avait proposé Kaolack, Podor, et Richard Toll. Finalement, la direction a retenu la cité sucrière, Richard Toll. Par ailleurs, cette période a coïncidé avec le départ à la retraite de mon père qui travaillait dans cette ville depuis des années à la Compagnie Sucrière Sénégalaise (CSS). Ça a été un moment fort pour moi, j'ai senti le poids qui pesait sur mes épaules, comme un passage de témoin.

\section{En 2013 vous avez commencé à travailler pour Radio Chine Internationale. De quand date cette radio et son implantation au Sénégal ?}

Ici, en Afrique, il y a seulement le bureau Sénégal à Dakar. Ce bureau de Dakar, qui est comme une station régionale, date en fait de 2015, mais effectivement, j'avais commencé à travailler pour eux dès 2013. C'était dans le cadre de Radio Chine Internationale ( $\mathrm{RCl})$ service français qui émettait une émission appelée "Fréquence Afrique » depuis Pékin17. Cela signifie que I'intervention était dans un pays francophone, mais il y a aussi Radio Chine service arabe ou Radio Chine service italien ou allemand, etc. Aujourd'hui, sur le continent, il y a aussi différentes antennes-relais qui font que la station est écoutée entre autres au Burundi, en République centrafricaine, etc. Il y a des pays où il y a des antennes-relais, et d'autres où $\mathrm{RCl}$ n'émet pas mais elle reste très diffusée à travers internet et dès 2013 j'administrais une de leurs pages

\footnotetext{
16 Le mouvement Moustarchidine, créé dans les années 1970 par Serigne Moustapha Sy, est issu de la confrérie Tidjiane, dont il se distingue notamment par une implication plus prononcée dans le champ politique.

17 II existe plusieurs travaux sur le rôle de Radio Chine en Afrique, voir Mihoubi Selma (2019), "La stratégie d'implantation de Radio Chine internationale (RCl) en Afrique sahélienne », Norois, 252, pp. 89-102. Pour une perspective historique depuis les années 1950, voir Morin-Allory Ronan (2011), « La Chine parle aux Africains. L'appareil médiatique de Pékin », Outre-Terre, 30, pp. 43-71.
} 
Facebook. II y a des émissions qui sont produites en Chine. Ainsi, parfois des journalistes africains qui allaient en Chine en profitaient pour enregistrer des émissions sur place, parce qu'il y a un service en français à Pékin. Quand on parle d'une radio appartenant à tel pays, les gens pensent qu'il y a des enjeux politiques derrière, mais nous, au niveau du bureau de la représentation, en tant que journalistes, quand nous proposons des thèmes, souvent ceux-ci sont liés à l'actualité et pas forcément à la Chine. Nous proposons des reportages, des émissions. II n'y a qu'une seule émission qui parle de la Chine pratiquement sur l'ensemble du programme, produit à Dakar je veux dire, et on ne nous impose rien. Même s'il y a aussi des émissions qui concernent les rapports entre la Chine et l'Afrique.

\section{Est-ce que c'était un choix de votre part, lié à une ligne éditoriale, de rentrer à Radio Chine Internationale?}

C'est un simple hasard. En 2013, j'étais en voyage et j'ai capté par hasard la radio. La radio date de 2010, mais je l'ai découverte et je me suis dit que c'était une radio qui m'intéressait, même si je ne connaissais pas de ressortissant chinois, ni même l'endroit où se situait le siège. Puis, un ami m'a mis en contact avec celui qui était là pour lancer la station radio, Rachid Tursun, qui se trouvait être le représentant de $R C /$ à Dakar. On est en février 2013, j'ai alors rencontré quelqu'un qui travaillait pour Xinhua - une des deux agences de presse nationale chinoise - et j'ai été accrédité au nom de $R C /$ pour couvrir le défilé de la fête de l'indépendance du Sénégal 2013. C'est ainsi que j'ai commencé à faire des reportages pour le siège de $R C l$, un reportage sur les wolofs, un autre sur le mariage traditionnel - « le mariage de Moussa » - pour l'émission "Fréquence Afrique » animée à l'époque par la belle voix de Sébastien, dit "Seb ». II y a eu aussi d'autres reportages filmés avec mon ami Yu Chen à Touba, le Magal de 2013, la panne du tuyau de Keur Momar Sarr qui avait privé d'eau toute la région de Dakar. J'écrivais aussi des articles pour le site de $R C l$. Un grand événement couvert pendant cette période a été la visite du président américain Barack Obama au Sénégal. En juin 2013, nous avons aussi reçu au bureau l'ambassadeur de la République populaire de Chine au Sénégal pendant cette période, Xia Huang.

\section{Dans le cadre de Radio Chine Internationale, vous avez aussi troqué votre casquette de reporter pour celle d'animateur. Vous vous occupez de deux émissions, une littéraire Printemps des écrivains, et l'autre sur l'histoire, Historama. Pourriez-vous nous parler de cette dernière, et déjà depuis quand avez-vous commencé à l'animer?}

Cela fait pratiquement 5 ans que j'anime Historama. C'est une émission hebdomadaire, donc il y a près de 200 émissions, je ne saurais même pas dire combien exactement. Parfois il y a des rediffusions ou des rétros mais ce sont chaque mois trois, quatre, cinq émissions qui sont réalisées. Maintenant, l'émission commence à être un peu connue dans le monde universitaire et les étudiants se passent le mot mais il y a plusieurs paramètres. Je dirais d'abord que les émissions ne sont pas dans l'espace académique. Les auditeurs ne sont pas que des historiens, ou des universitaires. II y a des gens qui sont justes intéressés par l'histoire et qui écoutent. C'est pourquoi quand on choisit les thèmes, c'est en fonction de plusieurs choses. On donne la parole aux témoins de l'histoire, à certains qui ont fait leur propres recherches. Parfois aussi 
dans les rencontres, littéraires ou à caractère historique, on tombe sur des gens qui sont intéressants et qui nous font découvrir un aspect du passé. Nous sommes preneurs de tous ceux qui regardent dans le rétroviseur et qui ont des choses à dire qui peuvent intéresser les auditeurs.

Une des premières émissions a été avec le grand poète Amadou Lamine Sall, coordonnateur du projet de mémorial de Gorée, et ancien secrétaire du très grand poèteprésident Léopold Sédar Senghor. C'est même sa voix qui est dans la bande annonce de l'émission. Amadou Lamine Sall m'a reçu dans son bureau à Dakar, siège du mémorial de Gorée. La première partie de l'interview était consacrée à la fondation mondiale pour la sauvegarde et le mémorial de Gorée dont il est le secrétaire général. II est revenu de long en large sur la nécessité d'ériger ce monument pour rendre hommage à toutes les mémoires souffrantes du monde et particulièrement la traite atlantique. Nous avons fait aussi une émission avec notre "frère » du Djoloff, Bouna Sémou Ndiaye, un des contributeurs pour la reconstitution du fond culturel du premier Festival mondial des arts nègres (FESMAN) de 1966. Il est aujourd'hui producteur et présentateur d'une émission de radio aux USA. Dans l'émission, il a raconté les étapes du rapatriement de ce patrimoine, notamment avec un film réalisé par les Soviétiques. II y a eu aussi Amadou Ndéné Ndaw, né en 1924, un haut responsable et spécialiste du travail à l'école qui m'a inspiré. II avait effectué une visite en Chine en 1960 en dirigeant une délégation des enseignants d'Afrique. II a raconté cette expérience dans Historama, c'est une rencontre qui m'a beaucoup marqué.

En fait, on est assez ouvert, l'émission concerne le Sénégal et la sous-région mais cela dépend aussi du thème et de l'invité. Quand on parle des guerres mondiales, on est plus généraliste. Il fut un moment où je travaillais sur l'apport des Africains et des Asiatiques, dans l'avènement d'un "monde libre ». Cela dépend aussi des invités, s'il y a des chercheurs étrangers qui sont de passage au Sénégal. Il y a eu par exemple des archéologues français qui sont venus parler de l'origine des mégalithes au Sénégal. Aussi, dernière chose, les réseaux sociaux nous ont permis beaucoup en termes d'interactions, pour des retours de la part d'auditeurs sur des émissions mais aussi parfois pour trouver des intervenants.

Martin Mourre

IMAF, Campus Condorcet (France)

\section{Bibliographie}

CAUDRON Fabrice, Grecos Myrto, JeAfar Ibtisseme (2018), « La formation des cadres des médias africains par le e-learning. Étude de cas d'un partenariat universitaire au niveau Master », @GRH, 26, pp. 79-102.

KANE Oumar (2008), "La FAD en Afrique francophone. Éléments historiques et enjeux récents 》, Distances et savoirs, 6, pp. 69-82.

Minoubı Selma (2019), "La stratégie d'implantation de Radio Chine internationale (RCl) en Afrique sahélienne », Norois, 252, pp. 89-102.

MoRIN-Allory Ronan (2011), "La Chine parle aux Africains. L'appareil médiatique de Pékin », OutreTerre, 30, pp. 43-71. 